Ann. Sci. forest., 1968, 25 (4), 237-249.

\title{
DES CARACTÉRISTIQUES NOUVELLES POUR L'ÉTUDE DES PROPRIÉTÉS MÉCANIQUES DES BOIS : LES COMPOSANTES DE LA DENSITÉ
}

\author{
R. KELLER \\ Station de Recherches sur la Qualité des Bois, \\ Centre national de Recherches forestières, 54 - Nancy \\ Institut national de la Recherche agronomique
}

\section{SOMMAIRE}

L'utilisation de courbes de variation de la densité favorise la mise en évidence de liaisons qui existent entre la structure du bois et sa résistance mécanique. Les mesures variées sur les courbes densitométriques apportent des renseignements qui peuvent être plus précis que ceux que donnent les mesures traditionnelles de largeur de cernes, de texture, ou de densité globale.

\section{1. - PRÉSENTATION DE LA MÉTHODE}

L'essai dont il est question comporte deux phases : d'une part, des mesures de résistance mécanique, et d'autre part, des mesures sur courbes densitométriques obtenues à partir de fragments prélevés sur les éprouvettes ayant subi les essais mécaniques. La comparaison des résultats et les calculs de corrélation permettent de comparer la valeur des informations fournies par les diverses mesures.

Les essais mécaniques ont porté sur 47 éprouvettes normalisées de $2 \times 2 \times 30\left({ }^{1}\right)$ de Douglas (humidité $8 \%$; toutes ont été tirées du même billon qui provenait de la forêt d'Orbey (Ht-Rhin). Malgré cette origine commune, on a pu observer des différences importantes d'aspect entre les éprouvettes (texture, proportion de bois de cœur et d'aubier, largeur de cernes) qui se sont d'ailleurs traduites dans les résultats obtenus.

Chaque éprouvette a été essayée en flexion statique sur une machine d'Amsler, les cernes étant tangentiels à l'effort, suivant le mode opératoire de la norme AFNOR B 51-008 :

- distance entre appuis : $28 \mathrm{~cm}$ (appuis cylindriques de $3 \mathrm{~cm}$ de diamètre),

- charge appliquée par un couteau cylindrique de $3 \mathrm{~cm}$ de diamètre au milieu de la face radiale, de façon que la flèche augmente d'environ $5 \mathrm{~mm} / \mathrm{mn}$.

( ${ }^{1}$ Les dimensions des éprouvettes sont données en $\mathrm{cm}$. 
Dans les parties intactes de chaque éprouvette, on a découpé deux éprouvettes de $2 \times 2 \times 6$, une de $2 \times 2 \times 2$ et une de $2 \times 2 \times 0,5$ pour la suite des mesures.

L'une des éprouvettes de $2 \times 2 \times 6$ a été utilisée pour l'étude de la compression axiale (norme AFNOR B 51-007), dans laquelle l'échantillon est comprimé entre les deux plateaux de la machine d'Amsler se rapprochant l'un de l'autre de façon que la charge augmente de 800 à $1200 \mathrm{~kg} / \mathrm{mn}$.

L'autre éprouvette de $2 \times 2 \times 6$ fut comprimée radialement (compression de flanc), jusqu'à ce que sa dimension radiale se soit abaissée du $1 / 3$ de sa valeur initiale. On a noté la charge maximum atteinte.

Finalement, l'ensemble des essais donne les résultats suivants :

- en flexion statique

- en compression axiale

: contrainte maximum en $\mathrm{kg} / \mathrm{cm}^{2}$,

flèche correspondante à cette charge,

valeur de la contrainte lorsque l'éprouvette a pris une flèche égale aux 3/100 de sa portée ;

— en compression de flanc : contrainte maximum en $\mathrm{kg} / \mathrm{cm}^{2}$.

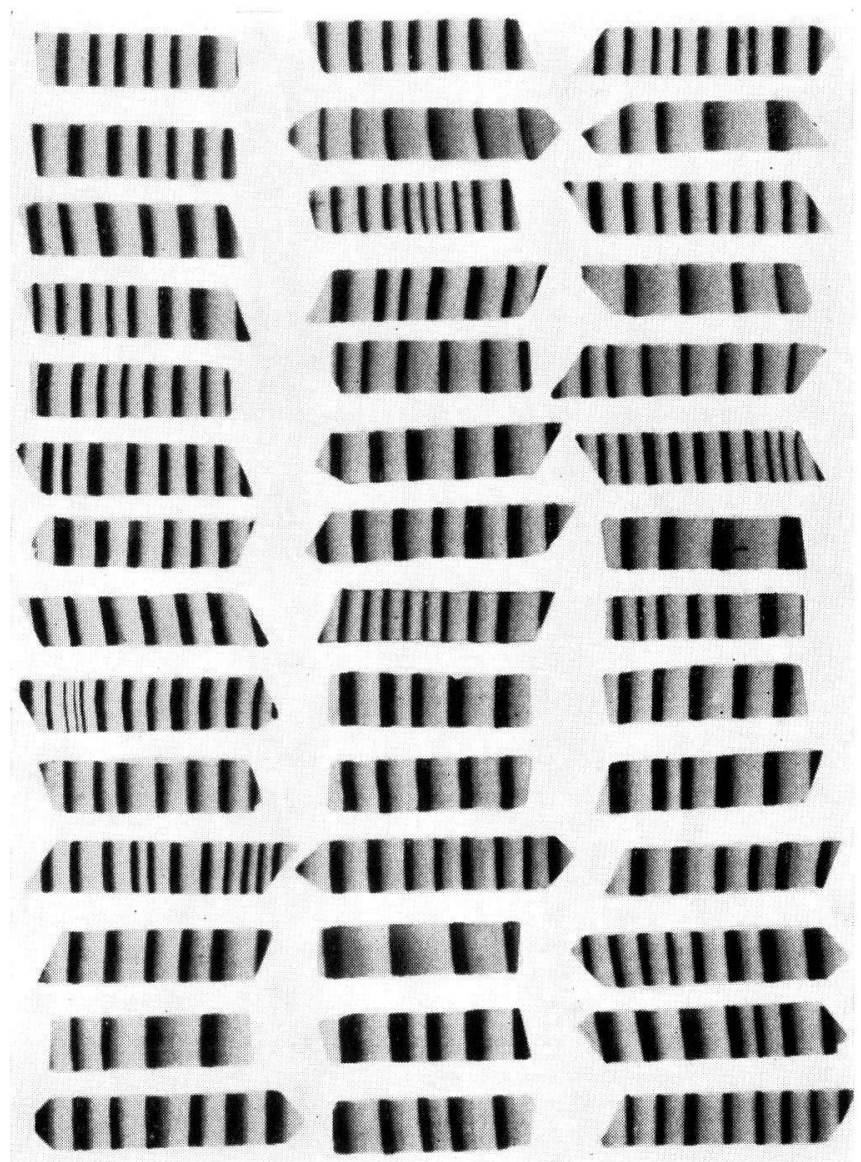

FIG. 1. - Photographie montrant la variabilité des éprouvettes utilisées pour l'analyse microdensitométrique

(Comme il s'agit du positif de la radiographie, le bois de printemps apparaît ici sous forme de zones claires et le bois d'été sous forme de zones sombres). 
La deuxième partie des essais s'est faite par la méthode, tout à fait différente, de l'étude en continu de la densité du bois. Les éprouvettes de $2 \times 2 \times 0,5$ ont servi à façonner de petits échantillons d'une épaisseur constante, égale à $5 \mathrm{~mm}$. Les deux autres dimensions : largeur et longueur valent respectivement environ 0,5 et $2 \mathrm{~cm}$. Les arêtes longitudinales de ces petits parallélipipèdes sont aussi perpendiculaires que possible aux limites annuelles des cernes.

Ces 47 éprouvettes fort peu encombrantes ont toutes été placées sur le même film radiographique nu et, selon la méthode classique du Laboratoire, irradiées pendant $9 \mathrm{mn}$ par des rayons X de grande longueur d'onde ; l'axe du rayonnement est parallèle au fil du bois et aux limites de cernes. Les rayons $\mathrm{X}$ sont plus ou moins arrêtés par le bois, selon qu'il est plus ou moins dense, et, après développement du film, les zones de faible densité (bois de printemps) apparaissent comme des surfaces sombres, et celles de forte densité comme des surfaces claires, l'ensemble donnant une représentation fidèle et continue de la nature de chaque cerne annuel (fig. 1).

Chaque image radiographique est analysée par un microdensitomètre enregistreur qui, en équilibrant l'intensité d'un faisceau lumineux traversant le film radiographique et celle d'un autre faisceau issu de la même source et passant à travers un coin optique, permet l'enregistrement continu de la variation de la densité du bois constituant chaque échantillon. Les courbes portent en abcisses les longueurs et en ordonnées les valeurs de la densité.

Ces courbes se prêtent à diverses mesures :

- largeur des cernes (obtenue avec précision grâce à la netteté du contraste sur la radiographie et le rapport de grandissement des ordonnées utilisé),

- valeur de la densité minimum année par année,

- valeur de la densité maximum année par année,

- pourcentage de la longueur radiale des cernes où l'on trouve une densité du bois supérieure à des valeurs choisies $\left(350,450,550\right.$ ou $\left.650 \mathrm{~g} / \mathrm{dm}^{2}\right)$.

Enfin, sur les cubes de $2 \times 2 \times 2$, on a mesuré le taux d'humidité du bois, le rapport de leurs poids anhydres à leurs volumes saturés (infradensité), et l'angle moyen des limites de cernes avec les faces radiales des éprouvettes.

Des comparaisons entre les résultats des essais mécaniques et des mesures sur radiographie d'une part, et des mesures classiques d'autre part, font ressortir l'intérêt des études aux rayons X faites en liaison avec les caractéristiques mécaniques des bois, d'autant plus que les mesures microdensitométriques sont couramment obtenues à partir de prélèvements non destructifs à la tarière de Pressler de $5 \mathrm{~mm}$ de diamètre.

Les variables explicatives sont au nombre de neuf :

$\mathrm{X}_{1}=$ largeur moyenne des cernes $(\mathrm{lm})$.

$\mathrm{X}_{2}=$ infradensité de l'échantillon déterminée sur les cubes de $2 \times 2 \times 2\left(\mathrm{P}_{0} / \mathrm{V}_{s}\right)$.

$\mathrm{X}_{3}=$ pourcentage de la longueur radiale de l'échantillon dont la densité est supérieure à $350 \mathrm{~g} / \mathrm{dm}^{3}$ $(\% d \geqslant 350)$.

$\mathrm{X}_{4}=$ pourcentage de la longueur radiale où la densité est supérieure à $450 \mathrm{~g} / \mathrm{dm}^{3}$.

$\mathrm{X}_{5}=$ pourcentage de la longueur radiale où la densité est supérieure à $550 \mathrm{~g} / \mathrm{dm}^{3}$.

$\mathrm{X}_{6}=$ pourcentage de la longueur radiale où la densité est supérieure à $650 \mathrm{~g} / \mathrm{dm}^{3}$.

$\mathrm{X}_{7}=$ moyenne des densités maxima $(\mathrm{dM})$.

$\mathrm{X}_{8}=$ écart entre la moyenne des densités maxima et la moyenne des densités minima $(\mathrm{dM}-\mathrm{dm})$. $\mathrm{X}_{9}=$ angle en degrés de la tangente aux cernes avec les faces radiales des éprouvettes.

Les sept données $\mathrm{X}_{1}, \mathrm{X}_{3}$ à $\mathrm{X}_{8}$ sont mesurées sur les courbes de variation de la densité en fonction du temps (fig. 2).

Par exemple :

$$
X_{3}=\left(\frac{b c}{a c}+\frac{d e}{c e}+\frac{f g}{e g}+\ldots\right) \times 100
$$

ac, ce, eg étant les largeurs de cernes de trois années successives.

$$
\mathrm{X}_{5}=\left(\frac{\mathrm{b}^{\prime} \mathrm{c}^{\prime}}{\mathrm{a}^{\prime} \mathrm{c}^{\prime}}+\frac{\mathrm{d}^{\prime} \mathrm{e}^{\prime}}{\mathrm{c}^{\prime} \mathrm{e}^{\prime}}+\frac{\mathrm{f}^{\prime} \mathrm{g}^{\prime}}{\mathrm{e}^{\prime} \mathrm{g}^{\prime}}+\ldots\right) \times 100
$$

avec $a^{\prime} c^{\prime}=a c, c^{\prime} e^{\prime}=c e, e^{\prime} g^{\prime}=e g$, les limites des cernes sur les graphiques étant quasi parallèles à l'axe des ordonnées. 


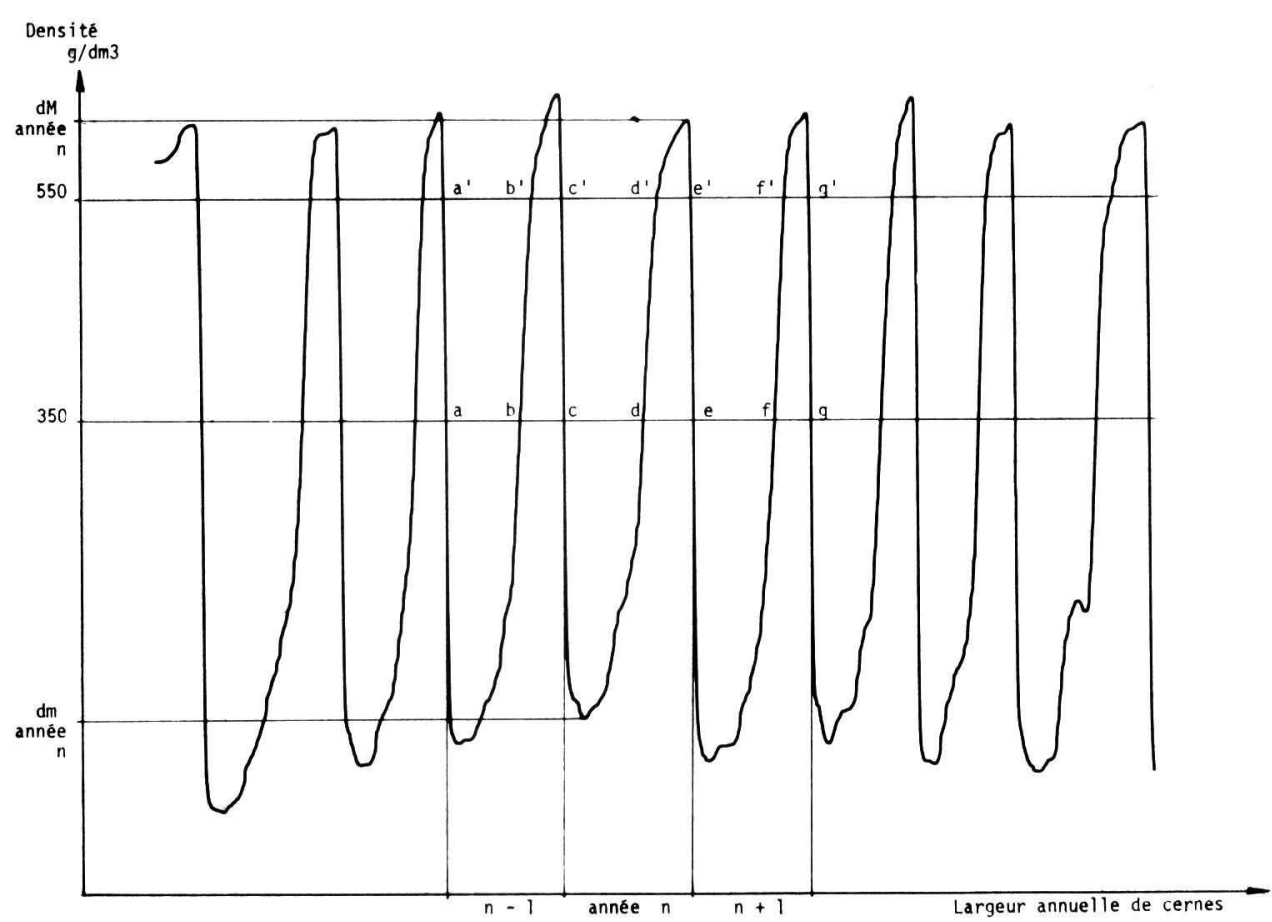

FIG. 2. - Courbe microdensitométrique d'un échantillon sur laquelle sont indiquées quelques composantes de la densité.

Les variables à expliquer sont les résultats des essais mécaniques :

$\mathrm{X}_{10}=$ contrainte à la rupture en flexion statique en $\mathrm{kg} / \mathrm{cm}^{2}$.

$\mathrm{X}_{11}=$ flèche correspondant à $\mathrm{X}_{10}$.

$\mathrm{X}_{12}=$ contrainte correspondant à une flèche de $8,4 \mathrm{~mm}$ (égale aux $3 / 100$ de la portée).

$\mathrm{X}_{13}=$ contrainte à la rupture en compression axiale en $\mathrm{kg} / \mathrm{cm}^{2}$.

$\mathrm{X}_{14}=$ contrainte maximum atteinte en compression de flanc.

\section{2. - RESULTATS}

Sur le tableau 1 sont portés les coefficients de corrélation entre les caractéristiques mécaniques et les variables explicatives.

Ce tableau montre l'intérêt de l'utilisation des caractéristiques nouvelles dont les coefficients de corrélation avec les variables à expliquer sont généralement les plus élevés, la résistance à la compression de flanc faisant exception à cette remarque.

Pour préciser l'étude du phénomène, on a recouru à la méthode de la régression progressive dans laquelle les variables sont introduites en fonction de l'importance de l'information supplémentaire qu'elles apportent à ce qu'une ou plusieurs autres variables ont déjà ( (expliqué ). La première variable introduite est celle qui a le plus fort coefficient de corrélation avec la variable à expliquer considérée. 


\section{TABLEAU 1}

Valeurs des coefficients de corrélation entre les caractéristiques mécaniques et les variables explicatives

\begin{tabular}{|c|c|c|c|c|c|c|c|c|c|}
\hline & $\operatorname{lm}_{X_{1}}$ & $\begin{array}{c}P_{0} / V_{s} \\
\mathrm{X}_{2}\end{array}$ & $\begin{array}{c}\% d \geqslant 350 \\
\mathrm{X}_{3}\end{array}$ & $\% \frac{d \geqslant 450}{\mathrm{X}_{4}}$ & $\begin{array}{c}\% d \geqslant 550 \\
X_{5}\end{array}$ & $\begin{array}{c}\% \\
\mathrm{X}_{6}\end{array}$ & $\begin{array}{l}\mathrm{dM} \\
\mathrm{X}_{7}\end{array}$ & $\mathrm{dm}$ & $\underset{\mathrm{X}_{9}}{\operatorname{angle}}$ \\
\hline Résistance en flexion statique $\left(\mathrm{X}_{10}\right) \ldots$ & $-0,55^{* *}$ & $0,52 * *$ & $0,65^{* *}$ & $0,70^{* *}$ & $0,78^{* *}$ & $0,64 * *$ & $0,77 * *$ & $-0,18$ & 0,09 \\
\hline Flèche maximum $\left(\mathrm{X}_{11}\right) \ldots \ldots \ldots \ldots$ & $-0,65 * *$ & 0,10 & $0,51^{* *}$ & $0,60 * *$ & $0,69 * *$ & $0,45 * *$ & $0,60 * *$ & $-0,37 * *$ & $-0,00$ \\
\hline Charge à la flèche de $8,4\left(\mathrm{X}_{12}\right) \ldots$ & $-0,57 * *$ & 0,10 & $0,54 * *$ & $0,59 * *$ & $0,63 * *$ & $0,41 * *$ & $0,51 * *$ & $-0,27$ & $-0,06$ \\
\hline Résistance en compression axiale $\left(\mathrm{X}_{13}\right)$ & $-0,23$ & $0,70 * *$ & $0,71^{* *}$ & $0,67 * *$ & $0,67^{* *}$ & $0,69 * *$ & $0,70 * *$ & $0,14^{* *}$ & 0,17 \\
\hline $\begin{array}{l}\text { Résistance en compression de flanc } \\
\left(\mathrm{X}_{14}\right) \ldots \ldots \ldots \ldots \ldots \ldots \ldots \ldots \ldots \ldots \ldots\end{array}$ & $0,29 *$ & 0,02 & $-0,16$ & $-0,23$ & $-0,18$ & 0,03 & $-0,07$ & 0,26 & $0,76^{* *}$ \\
\hline
\end{tabular}

$\operatorname{lm}=$ largeur moyenne des cernes $\left(\mathrm{X}_{1}\right)$,

$P_{0} / V_{s}=$ infradensité, rapport du poids anhydre d'un échantillon à son volume à l'état saturé $\left(\mathrm{X}_{2}\right)$,

Caractéristiques nouvelles $\begin{cases}\% d \geqslant x & =\text { pourcentage de longueur radiale où la densité du bois égale ou dépasse la densité de } x \mathrm{~g} / \mathrm{dm}^{3}\left(\mathrm{X}_{3} \text { à } \mathrm{X}_{6}\right), \\ \mathrm{dM} & =\text { densité maximum }\left(\mathrm{X}_{7}\right), \\ \mathrm{dm} & =\text { densité minimum, }\end{cases}$

angle $=$ angle de la tangente aux cernes avec les faces radiales de l'éprouvette $\left(\mathrm{X}_{\mathbf{9}}\right)$,

** $\quad=$ seuil de signification à $1 \%$,

* $\quad=$ seuil de signification à $5 \%$. 
La densité minimum semblant, d'après ce tableau, n'avoir qu'une importance restreinte, on a introduit dans la régression comme nouvelles variables explicatives $\mathrm{X}_{1}$ à $\mathrm{X}_{7}, \mathrm{X}_{9}$ et l'écart $\mathrm{X}_{8}$ entre les moyennes des densités maximales et les moyennes des densités minimales. On s'en est tenu à $\mathrm{dM}$ et $\mathrm{dM}-\mathrm{dm}$ pour éviter d'introduire d'autres combinaisons linéaires entre ces variables. Cependant, on verra que la densité minimum peut pratiquement apparaître seule dans les résultats (cas de la flèche correspondant à la charge maximum atteinte en flexion statique).

\section{1. - Résistance en flexion statique}

Les carrés des coefficients de corrélation totale, ou les pourcentages de la variation de cette caractéristique ( expliquée ) par les variables mesurées sont les suivants :

\begin{tabular}{c|c|c|c|c|c|c|c|c|c} 
& $\mathrm{X}_{1}$ & $\mathrm{X}_{2}$ & $\mathrm{X}_{3}$ & $\mathrm{X}_{4}$ & $\mathrm{X}_{5}$ & $\mathrm{X}_{6}$ & $\mathrm{X}_{7}$ & $\mathrm{X}_{8}$ & $\mathrm{X}_{9}$ \\
\hline$\%$ & 30,2 & 27 & 42,2 & 49 & 60,9 & 41 & 59,3 & 67,2 & 0
\end{tabular}

La méthode de l'analyse de régression progressive conduit à choisir $\mathrm{X}_{8}$, écart entre $\mathrm{dM}$ et $\mathrm{dm}$, comme la variable explicative ayant le plus d'importance au niveau considéré.

Son coefficient de corrélation multiple atteint 0,823 , et la première équation de régression s'écrit :

$$
\mathrm{X}_{10}\left(\mathrm{en} \mathrm{kg} / \mathrm{cm}^{2}\right)=0,1445 \mathrm{X}_{8}-29,1040
$$

L'introduction d'une nouvelle variable permet d'améliorer légèrement le coefficient de corrélation multiple $(0,833)$ et de diminuer l'écart-type résiduel. Il s'agit du pourcentage $\mathrm{X}_{5}$ de longueur radiale totale où le bois formé a une densité supérieure ou égale à $550 \mathrm{~g} / \mathrm{dm}^{3}$, et l'équation prend la forme :

$$
\mathrm{X}_{10}\left(\mathrm{en} \mathrm{kg} / \mathrm{cm}^{2}\right)=0,1006 \mathrm{X}_{8}+0,2349 \mathrm{X}_{5}-13,5786
$$

La prise en considération d'autres variables améliorerait encore un peu les équations de régression, mais le gain est trop faible pour qu'il soit utile d'augmenter le nombre des grandeurs explicatives.

Tout se passe comme si cette résistance était surtout le fait du bois qui a une forte densité $\left(d \geqslant 550 \mathrm{~g} / \mathrm{dm}^{3}\right)$. La figure 3 montre la liaison entre cette caractéristique mécanique et l'écart entre la densité maximum et la densité minimum.

\section{2. - Flèche atteinte à la charge maximum en flexion statique}

Les pourcentages de la variation expliquée de cette caractéristique sont portés ci-dessous :

\begin{tabular}{c|c|c|c|c|c|c|c|c|c} 
& $\mathrm{X}_{1}$ & $\mathrm{X}_{2}$ & $\mathrm{X}_{3}$ & $\mathrm{X}_{4}$ & $\mathrm{X}_{5}$ & $\mathrm{X}_{6}$ & $\mathrm{X}_{7}$ & $\mathrm{X}_{8}$ & $\mathrm{X}_{9}$ \\
\hline$\%$ & 42,2 & 0 & 26 & 36 & 47,6 & 20,2 & 36 & 49 & 0
\end{tabular}




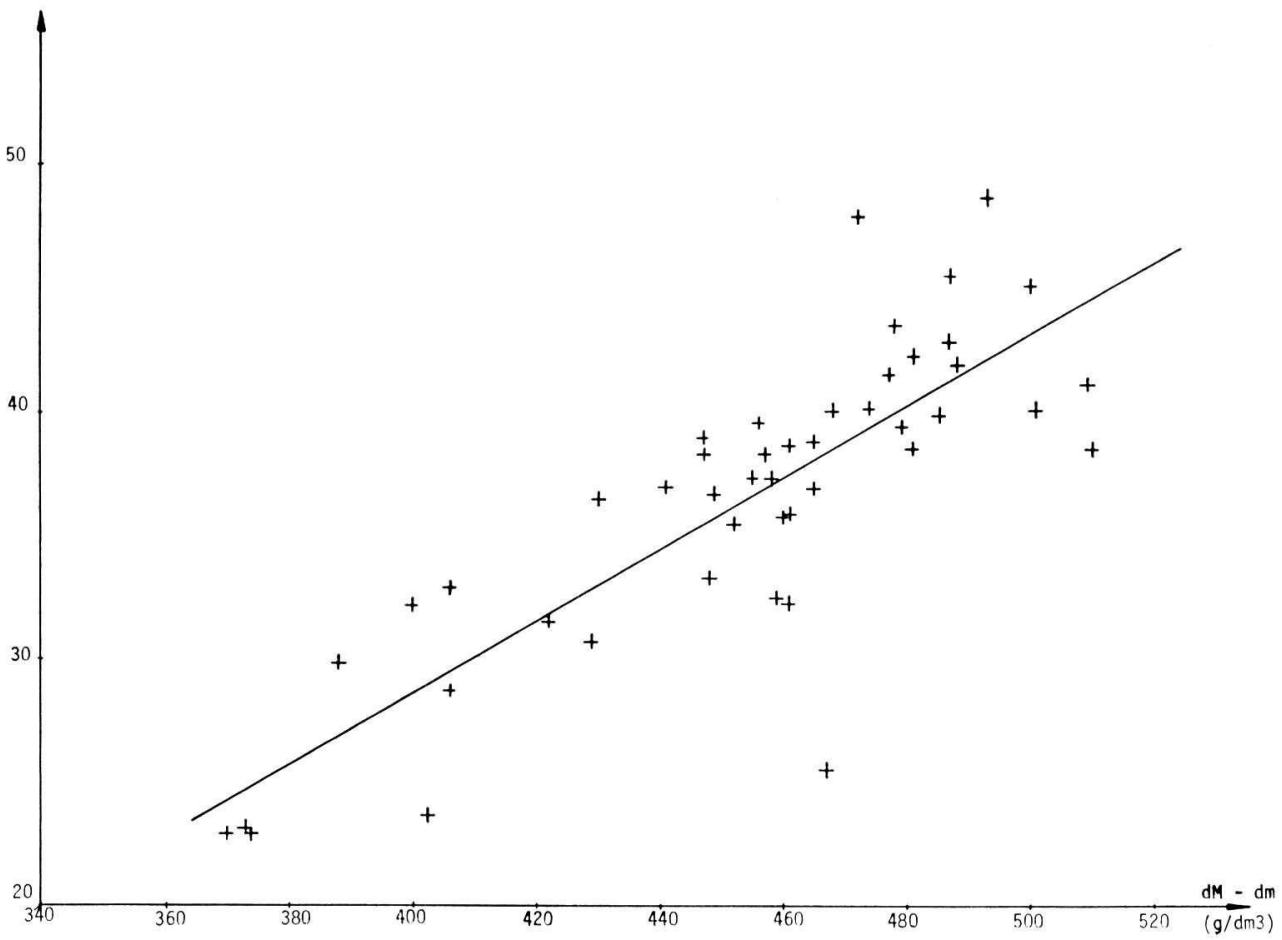

FIG. 3. - Liaison entre ia résistance en flexion statique et l'écart entre la densité maximum et la densité minimum $(r=0,823)$.

A ce niveau, la meilleure caractéristique explicative est encore l'écart $\mathrm{X}_{8}$ entre la densité maximum et la densité minimum, mais le pourcentage de variation (c expliquée $), 49 \%$, peut être amélioré si l'on recourt, dans l'ordre, à quatre autres variables explicatives :

- densité maximum $\left(\mathrm{X}_{7}\right)$.

- largeur moyenne des cernes $\left(\mathrm{X}_{1}\right)$.

- pourcentage de longueur radiale où le bois a une densité égale ou supérieure à $650 \mathrm{~g} / \mathrm{dm}^{3}\left(\mathrm{X}_{6}\right)$.

- pourcentage de longueur radiale où le bois a une densité égale ou supérieure à $550 \mathrm{~g} / \mathrm{dm}^{3}\left(\mathrm{X}_{5}\right)$.

Le coefficient de corrélation multiple atteint alors 0,863 . Si l'on poussait plus loin, les tests de STUDENT, ou rapports des coefficients de régression à leur écarttype, à chaque nouveau palier d'introduction de variables, ne seraient plus significatifs. 
Au cinquième palier, l'équation de régression pourra s'écrire : $\mathrm{X}_{11}($ en $\mathrm{mm})=0,0723 \mathrm{X}_{8}-0,0660 \mathrm{X}_{7}-0,6304 \mathrm{X}_{1}+0,1539 \mathrm{X}_{6}-0,0345 \mathrm{X}_{5}+29,7633$

Dans cette équation, l'influence de la densité maximum est faible puisque les coefficients qui affectent cette variable sont du même ordre de grandeur en valeur absolue, mais de signes contraires.

En effet :

$X_{8}=d M-d m, X_{7}=d M$ et $0,723(d M-d m)-0,0660 d M$ est très voisin de $-0,0723 \mathrm{dm}$ lorsqu'on néglige $0,0063 \mathrm{dM}$.

Au palier numéro 6 , le coefficient final de $\mathrm{d} M$ diminue encore et ne vaut plus que 0,0003 .

Ceci traduit le fait que la flèche correspondant à la charge maximum sera d'autant plus faible que la densité minimum et la largeur moyenne des cernes seront plus grandes. Les coefficients de corrélation totale entre la flèche d'une part, la densité minimum et la largeur moyenne des cernes d'autre part, valent respectivement $-0,37$ et $-0,65$ et traduisent significativement cette tendance.

Il semble que cette caractéristique nécessite, pour être élevée, à la fois la présence de bois à forte densité $\left(\mathrm{d} \geqslant 650 \mathrm{~g} / \mathrm{dm}^{3}\right)$ donnant à l'éprouvette la résistance, et celle de bois à faible densité capable de se déformer et conférant de l'élasticité à l'ensemble.

\section{3. - Résistance en compression axiale}

Les pourcentages de variation "(expliquée ) font ressortir l'importance des variables explicatives se rapportant aux différents niveaux de densité :

\begin{tabular}{l|l|l|l|l|l|l|l|l|l} 
& $\mathrm{X}_{1}$ & $\mathrm{X}_{2}$ & $\mathrm{X}_{3}$ & $\mathrm{X}_{4}$ & $\mathrm{X}_{5}$ & $\mathrm{X}_{6}$ & $\mathrm{X}_{7}$ & $\mathrm{X}_{8}$ & $\mathrm{X}_{9}$ \\
\hline$\%$ & 5,3 & 49 & 50,4 & 44,9 & 44,9 & 47,6 & 49 & 44,9 & 2,9
\end{tabular}

Au premier palier apparaît le pourcentage $\mathrm{X}_{3}$ de longueur radiale des éprouvettes où la densité du bois égale ou dépasse $350 \mathrm{~g} / \mathrm{dm}^{3}$; le coefficient de corrélation multiple vaut 0,714 à ce niveau.

L'introduction de l'infradensité $\mathrm{X}_{2}$, puis du pourcentage $\mathrm{X}_{6}$ de longueur radiale où la densité du bois égale ou dépasse $650 \mathrm{~g} / \mathrm{dm}^{3}$ le fait progresser significativement jusqu'à 0,831 . On pourra s'en tenir à l'équation de régression :

$$
\mathrm{X}_{13}\left(\mathrm{en} \mathrm{kg} / \mathrm{cm}^{2}\right)=1,3226 \mathrm{X}_{3}+1,1550 \mathrm{X}_{2}+5,0332 \mathrm{X}_{6}+51,7507
$$

Les faibles densités semblent être, dans ce type d'essai, le facteur limitant. Les éprouvettes cèdent d'autant plus facilement que la proportion de bois à faible résistance mécanique $\left(\mathrm{d} \leqslant 350 \mathrm{~g} / \mathrm{dm}^{3}\right)$ est plus grande.

\section{4. - Résistance en compression de flanc}

Les pourcentages de variation ( expliquée ) mettent en évidence au premier palier l'importance, danc ce type d'essai, de l'angle de la tangente aux cernes avec 
les faces radiales de l'éprouvette, l'effort en compression de flanc s'exerçant radialement (fig. 4) :

\begin{tabular}{|c|c|c|c|c|c|c|c|c|c|}
\hline & $\mathrm{X}_{1}$ & $\mathrm{X}_{2}$ & $\mathrm{X}_{3}$ & $\mathrm{X}_{4}$ & $X_{5}$ & $\mathrm{X}_{6}$ & $X_{7}$ & $\mathrm{X}_{8}$ & $X_{9}$ \\
\hline$\%$ & 8,4 & 0 & 2,6 & 5,3 & 3,2 & 0 & 0 & 1,7 & 57,7 \\
\hline
\end{tabular}

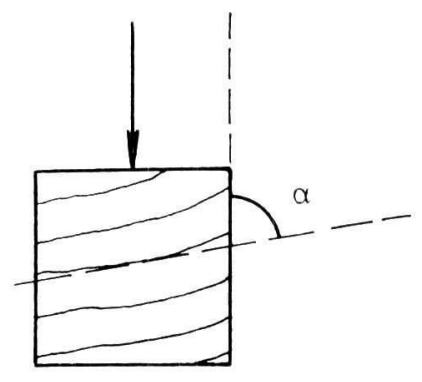

FIG. 4. - Mesure de l'angle $\alpha$ formé par la face radiale et la tangente aux cernes. Sens d'application de la force de compression sur l'éprouvette

Cet angle $\mathrm{X}_{9}$ et la résistance à la compression de flanc $\mathrm{X}_{14}$ sont liés par un coefficient de corrélation de 0,764 ; c'est la raison pour laquelle il est recommandé de n'utiliser dans ces essais que des éprouvettes où la tangente aux cernes soit perpendiculaire aux faces radiales.

Au palier suivant, apparaît le pourcentage $\mathrm{X}_{5}$ de longueur radiale où la densité $\mathrm{du}$ bois égale ou dépasse $550 \mathrm{~g} / \mathrm{dm}^{3}$.

Pour un angle donné, cette résistance tend à diminuer lorsque $\mathrm{X}_{5}$ augmente. Le coefficient de corrélation entre $\mathrm{X}_{5}$ et $\mathrm{X}_{14}$ vaut $-0,18$, mais n'est pas significatif. Ces deux variables ( expliquent ) $68 \%$ de la variation. Si l'on introduit l'infradensité $\mathrm{X}_{2}$, puis le pourcentage $\mathrm{X}_{6}$ de longueur radiale où la densité égale ou dépasse $650 \mathrm{~g} / \mathrm{dm}^{3}$, le pourcentage d'explication atteint $72 \%$. Pour écrire l'équation de régression, il est bon de s'en tenir à trois variables :

$$
\mathrm{X}_{14}\left(\mathrm{en} \mathrm{kg} / \mathrm{cm}^{2}\right)=0,7768 \mathrm{X}_{9}-0,7515 \mathrm{X}_{5}+0,1697 \mathrm{X}_{2}-41,4415
$$

le coefficient de corrélation valant 0,841 .

On peut signaler qu'aux huitième et neuvième paliers, où s'introduisent respectivement la densité maximum $X_{7}$ et l'écart $X_{8}$ entre la densité maximum et la densité minimum, l'influence de $\mathrm{X}_{7}$, par le jeu des coefficients de régression, devient faible devant celle de la densité minimum. $\mathrm{X}_{14}$ aurait tendance à augmenter quand la densité minimum augmente (coefficient de corrélation non significatif : 0,26).

Il paraît normal que l'angle des faces des cernes avec la direction de la force appliquée joue un grand rôle dans les résultats. Plus $\alpha$ est proche de $45^{\circ}$, plus un phé- 
TABLEAU 2

Tableau récapitulatif de l'analyse de régression

\begin{tabular}{|c|c|c|c|c|c|c|c|c|c|}
\hline \multirow[b]{2}{*}{ Résistance en flexion statique $\left(\mathrm{X}_{10}\right)$} & 1 & 2 & 3 & 4 & 5 & 6 & 7 & 8 & 9 \\
\hline & $\begin{array}{l}\mathrm{dM}-\mathrm{dm} \\
0,823^{* * *}\end{array}$ & $\begin{array}{l}\% d \geqslant 550 \\
0,833\end{array}$ & $\begin{array}{r}\mathrm{dM} \\
0,838\end{array}$ & $\begin{array}{c}\% d \geqslant 450 \\
0,842\end{array}$ & $\begin{array}{l}\% d \geqslant 350 \\
0,853\end{array}$ & $\begin{array}{l}\text { angle } \\
0,863\end{array}$ & $\begin{array}{c}\% d \geqslant 650 \\
0,866\end{array}$ & $\begin{array}{c}P_{0} / V_{s} \\
0,868\end{array}$ & $\operatorname{lm}_{0,868}$ \\
\hline Flèche maximum $\left(\mathrm{X}_{11}\right) \ldots \ldots \ldots \ldots$ & $\begin{array}{l}\mathrm{dM}-\mathrm{dm} \\
0,700^{* * *}\end{array}$ & $\begin{array}{c}\mathrm{dM} \\
0,759 * *\end{array}$ & $\operatorname{lm}_{0,822 * * *}$ & $\begin{array}{c}\% d \geqslant 550 \\
0,838\end{array}$ & $\begin{array}{l}P_{0} / V_{s} \\
0,863^{*}\end{array}$ & $\begin{array}{c}\% d \geqslant 650 \\
0,865\end{array}$ & $\begin{array}{c}\% d \geqslant 450 \\
0,869\end{array}$ & $\begin{array}{c}\text { angle } \\
0,873\end{array}$ & $\begin{array}{l}o d \geqslant 350 \\
0,879\end{array}$ \\
\hline Charge à la flèche de $8,4\left(\mathrm{X}_{12}\right) \ldots \ldots$ & $\begin{array}{l}\% d \geqslant 550 \\
0,638 * * *\end{array}$ & $\begin{array}{c}P_{0} / V_{s} \\
0,723^{* *}\end{array}$ & $\operatorname{lm}_{0,736}$ & $\begin{array}{c}\% d \geqslant 350 \\
0,749\end{array}$ & $\begin{array}{c}\mathrm{dM} \\
0,757\end{array}$ & $\begin{array}{c}\mathrm{d} M-\mathrm{dm} \\
0,772\end{array}$ & $\begin{array}{c}\% d \geqslant 450 \\
0,776\end{array}$ & $\begin{array}{l}\text { angle } \\
0,788\end{array}$ & $\begin{array}{c}\% d \geqslant 650 \\
0,788\end{array}$ \\
\hline Résistance en compression axiale $\left(\mathrm{X}_{13}\right)$ & $\begin{array}{l}\% d \geqslant 350 \\
0,714^{* * *}\end{array}$ & $\begin{array}{c}P_{0} / V_{s} \\
0,784 * *\end{array}$ & $\begin{array}{l}\% d \geqslant 650 \\
0,831 * *\end{array}$ & $\begin{array}{l}\mathrm{dM} \\
0,836\end{array}$ & $\begin{array}{l}\% d \geqslant 550 \\
0,846\end{array}$ & $\operatorname{lm}_{0,848}$ & $\begin{array}{c}\% d \geqslant 450 \\
0,851\end{array}$ & $\begin{array}{c}\mathrm{dM}-\mathrm{dm} \\
0,853\end{array}$ & $\begin{array}{c}\text { angle } \\
0,854\end{array}$ \\
\hline $\begin{array}{l}\text { Résistance en compression de flanc } \\
\qquad\left(\mathrm{X}_{14}\right) \ldots \ldots \ldots \ldots \ldots \ldots \ldots \ldots \ldots\end{array}$ & $\underset{0,764 * * *}{\text { angle }}$ & $\begin{array}{l}\% d \geqslant 550 \\
0,824^{* *}\end{array}$ & $\begin{array}{l}P_{0} / V_{s} \\
0,841^{*}\end{array}$ & $\begin{array}{c}\% d \geqslant 650 \\
0,849\end{array}$ & $\operatorname{lm}_{0,852}$ & $\begin{array}{c}\% d \geqslant 450 \\
0,861\end{array}$ & $\begin{array}{l}\% d \geqslant 350 \\
0,866\end{array}$ & $\begin{array}{l}\mathrm{dM} \\
0,873\end{array}$ & $\underset{0,886^{*}}{\mathrm{dM}-\mathrm{dm}}$ \\
\hline \multicolumn{5}{|c|}{$\begin{aligned}= & \text { largeur moyenne des cernes }\left(\mathrm{X}_{1}\right) \\
= & \text { infradensité, rapport du poids anhydre d'un échantillon à son } \\
& \text { volume saturé }\left(\mathrm{X}_{2}\right) \\
= & \text { pourcentage de longueur radiale où la densité du bois égale ou } \\
& \text { dépasse } x \mathrm{~g} / \mathrm{dm}^{3}\left(\mathrm{X}_{3} \text { à } \mathrm{X}_{6}\right)\end{aligned}$} & $\begin{array}{l}\mathrm{dM} \\
\mathrm{dm} \\
\mathrm{dM}-\mathrm{dm} \\
\text { angle }\end{array}$ & \multicolumn{4}{|c|}{$\begin{aligned}= & \text { densité maximum }\left(\mathrm{X}_{7}\right) \\
= & \text { densité minimum } \\
= & \text { écart de densités }\left(\mathrm{X}_{8}\right) \\
= & \text { angle de la tangente aux cernes avec les faces } \\
& \text { radiales de l'éprouvette }\left(\mathrm{X}_{9}\right) .\end{aligned}$} \\
\hline $\begin{array}{l}\text { *** }: \text { seuil de signification à } 1 \% \\
\text { ** } \quad \text { : seuil de signification à } 1 \% \\
\text { * } 1 \text { seuil de signification à } 5 \%\end{array}$ & palier c & $\begin{array}{l}\text { idéré de } 1 \\
\text { déré de } 1 \text {, }\end{array}$ & coduction & chaq & $\begin{array}{l}\text { uvelle vat } \\
\text { ivelle var }\end{array}$ & le. & & & \\
\hline
\end{tabular}

Les variables explicatives situées à gauche des traits verticaux sont celles qui ont servi à écrire les équations de régression relatives aux cinq variables expliquées. 
nomène de glissement entre les couches annuelles est susceptible de se produire. Il semble plus difficile d'expliquer les différences dans le rôle des divers niveaux de densité $(d \geqslant 550$ en corrélation négative et $d \geqslant 650$ en corrélation positive).

La figure 5 représente la liaison existant entre cette résistance et l'angle exprimé en degrés.

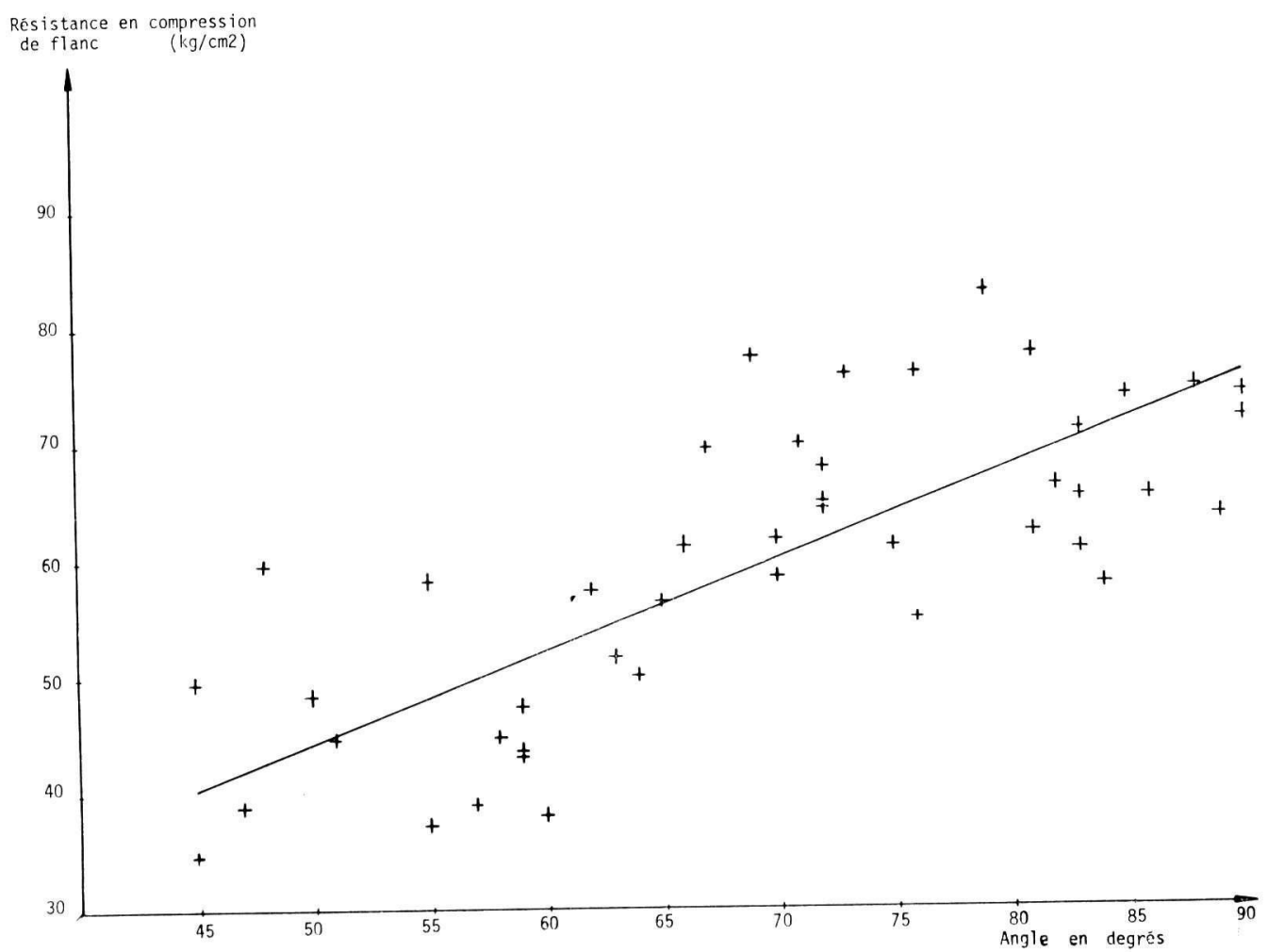

FIG. 5. - Liaison entre la résistance en compression de flanc et l'angle $\alpha(r=0,764)$.

Le tableau 2 résume les résultats obtenus. Les chiffres surmontant les colonnes représentent l'ordre d'apparition de chaque variable dans l'étude de la régression progressive qui se rapporte à la caractéristique mécanique envisagée. Dans chaque case sont portées la nature de la variable explicative et la valeur du coefficient de corrélation multiple, compte tenu du fait que les variables explicatives écrites à gauche de cette case sont déjà intervenues dans le calcul de régression.

\section{CONCLUSION}

Les composantes de la densité, caractéristiques nouvelles facilement mises en évidence et mesurées grâce à la méthode microdensitométrique, sont plus satisfaisantes dans l'étude des caractéristiques mécaniques du Douglas que les variables explicatives généralement utilisées jusqu’à présent. 
Au lieu de prendre la texture seule, qui correspond à la définition de MORK, et dont les mesures au microscope peuvent être l'objet d'interprétations délicates, on a utilisé une gamme de grandeurs analogues correspondant à diverses densités et facilement mesurables.

Par exemple, les coefficients de corrélation entre la résistance en flexion statique d'une part, et chacune de ces grandeurs d'autre part, varient sensiblement :

\begin{tabular}{|c|c|c|c|c|}
\hline & $\% d \geqslant 350$ & $\% d \geqslant 450$ & $\% \mid d \geqslant 550$ & $\% \mid d \geqslant 650$ \\
\hline $\begin{array}{l}\text { Coefficient de corrélation avec résistance en fle- } \\
\quad \text { xion statique } \ldots \ldots \ldots \ldots \ldots \ldots \ldots \ldots \ldots \ldots\end{array}$ & 0,65 & 0,70 & 0,78 & 0,65 \\
\hline
\end{tabular}

Mis à part le cas de la résistance en compression de flanc, où c'est l'angle de la tangente aux cernes avec les faces radiales des éprouvettes qui apparaît en premier lieu dans la régression, ce sont les composantes de la densité qui prennent la première, ou même les deux premières places dans les études de régression, devant les variables explicatives traditionnelles.

Les résultats obtenus et les tentatives d'explication avancées demanderaient à être vérifiés, notamment par l'essai d'éprouvettes artificielles formées de couches de bois collées où l'on ferait varier l'importance relative des zones à forte ou à faible densité, en choisissant convenablement les essences constituant les diverses couches.

L'utilisation des composantes de la densité dans le domaine de la technologie, comme dans bien d'autres d'ailleurs, est une méthode d'analyse dont les résultats sont très encourageants, et d'autant plus intéressants qu'elle peut être appliquée à l'étude des utilisations futures d'arbres sur pied, à partir de prélèvements non destructifs à la tarière de Pressler de $5 \mathrm{~mm}$ de diamètre.

Reçu pour publication en juillet 1968

\section{REMERCIEMENTS}

Nous remercions la Station de Biométrie du CNRF-INRA, et en particulier C. Millier, qui se sont chargés des calculs statistiques de cette étude.

\section{SUMMARY}

NeW CHARACTERISTICS FOR THE STUdy OF THE MECHANICAL PROPERTIES OF WOOD : DENSITY COMPONENTS

Two different kinds of test have been parallelly carried out : on the other hand, several criteria of mechanical strength have been determined, using parallelepiped normalized samples; on the other hand microdensitometric measurements of wood density components have been made on X-ray photographs of specimen taken from these samples. The correlations between strength and density components are narrow (table $\left.n^{\circ} 1\right)$, and furthermore the progressive regression analysis $\left(\operatorname{table} n^{\circ} 2\right)$ show that the latter are better than traditional data (ringwidth, percentage of latewood and specific gravity) to explain mẹchanical results, 


\section{ZUSAMMENFASSUNG}

\section{Neue Merkmale zum studium der mechanischen Eigenschaften des Holzes : DIE DiCHTEK.OMPONENTEN}

Es wurde versucht, zwischen zwei Messverfahren eine Parallele zu ziehen : einerseits Messungen der Druck - und Biegefestigkeit an Holzproben und andererseits mikrodensitometrischen Messungen der «Dichtekomponenten » des Holzes and Hand einer Röntgenstrahlenaufnahme von Teilchen derselben Holzproben. Die Korrelationskoefficiente (Tabelle 1) zwischen den verschiedenen mechanischen Festigkeiten und den Komponenten sind höher als diejenigen zwischen den mechanischen Festigkeiten und den herkömmlichen Merkmalen (Anteil des Spätholzes, Breite der Jahresringe, Dichte). Deshalb eignen sich die «Dichtekomponenten » am besten um den beobachteten Erscheinungen näher zu kommen (vgl. die Regressionsergebnisse der Tabelle 2).

\section{RÉFÉRENCES BIBLIOGRAPHIQUES}

Polge H., 1966. Etablissement des courbes de variation de la densité du bois par exploration densitométrique de radiographies d'échantillons prélevés à la tarière sur des arbres vivants. Applications dans les domaines technologique et physiologique. Thèse Doctorat ès Sciences.

TOMAssone R., 1965. L'analyse de régression progressive. Note interne CNRF.

Tomassone R. et Millier C., 1968. Eléments d'analyse statistique à plusieurs variables. Chap. 2. $\mathrm{La}$ régression. Station de Biométrie. Note interne CNRF.

VENET J., 1953. Etude de la résistance mécanique des bois de mine en fonction des facteurs de la production forestière. Thèse Univ. Nancy. 